\title{
Evaluation of EIT System Performance
}

\author{
Yasheng Maimaitijiang ${ }^{1}$, Stephan Böhm² ${ }^{2}$ Pascal O. Gaggero², \\ Andy Adler ${ }^{1,2}$ \\ ${ }^{1}$ Systems and Computer Engineering, Carleton University, Ottawa, Canada \\ ${ }^{2}$ Centre suisse d'electronique et de Microtechnique (CSEM), Landquart, Switzerland \\ E-mail: \{mamatjan,adler\}@sce.carleton.ca, \\ \{stephan.boehm, pascal.gaggero\}@csem.ch
}

\begin{abstract}
An electrical impedance tomography (EIT) system images internal conductivity from surface electrical stimulation and measurement. Such systems necessarily comprise multiple design choices from cables and hardware design to calibration and image reconstruction. In order to compare between EIT systems and to study the consequences of changes in system performance, this paper describes a systematic approach to evaluate the performance of the EIT systems. The system to be tested is connected to a saline phantom in which calibrated contrasting test objects are systematically positioned using a position controller. A set of evaluation parameters are proposed which characterize: i) data and image noise, ii) data accuracy, iii) detectability of single contrasts and distinguishability of multiple contrasts, and iv) accuracy of reconstructed image (amplitude, resolution, position and ringing). Using this approach, we evaluate three different EIT systems and illustrate the use of these tools to evaluate and compare performance. In order to facilitate use of this approach, all details of the phantom, test objects and position controller design are made publicly available including the source code of the evaluation and reporting software.
\end{abstract}

Keywords: Electrical Impedance Tomography, Calibration, Physical Phantom

\section{Introduction}

Electrical impedance tomography (EIT) is a non-invasive imaging technique in which changes in electrical conductivity within a body are visualized using current injections and voltage readings via an array of electrodes arranged around a body. EIT has been proposed for use in geophysics, process and physiological monitoring. One promising application of EIT is the monitoring of ventilation in patients to determine the distribution of breathing gases, to discriminate normally aerated from collapsed or overdistended lung tissue (Victorino et al 2004, Frerichs et al 2003).

In order to be able to correctly interpret these clinical EIT images it is essential to know the performance characteristics of the EIT system used. While many approaches have been proposed to measure isolated aspects of system performance, the goal of this paper is to present a systematic methodology to evaluate the performance of complete EIT systems, including the electrode interface, instrumentation hardware, and image 
reconstruction. We broadly classify such tests in categories of: measures of accuracy, and measures of detectability. Measures of accuracy are the amplitude response, resolution and position error, answering questions as to whether the test objects are represented correctly. The measures of detectability address the question of minimum size and conductivity contrast for the objects to be detected.

Two types of EIT phantoms have been proposed: physical and electrical mesh phantoms (Rigaud and Morucci 1996). Mesh phantoms are constructed from electrical components, generally using a combination of resistors and capacitors (Griffiths 1988, Griffiths 1995, Hahn et al 2000, Hahn et al 2008, Gagnon and Guardo 2005, Schneider et al 2000). These elements are interconnected in particular topologies as in the Cardiff phantom (Griffiths 1988), the wheel phantom (Griffiths 1995) or the Göttingen phantoms (Hahn et al 2000, Hahn et al 2008). Recently, a finite element model (FEM) based resistive mesh phantom with many resistors was proposed by Gagnon et al (2010) to provide a more accurate system representation. However, mesh phantoms are limited by the resolution of the FEM and resistor mesh, the cost and manufacturing time associated with a large number of high precision resistors.

Physical phantoms are based on a conductive medium with conductivity contrasting targets; the most common model is a saline filled non-conductive tank with conductive electrodes on the boundary. A recent comparison of physical phantoms is provided by Oh et al (2008). Many materials have been used for conductivity targets, including: acrylic plastics, polyacrylamide, stainless steel, TX151, agar, animal hide gelatine, and vegetables. One key limitation of the physical phantoms is that the conductivity changes with time due to the evaporation of water, ionic diffusion or biological decay of organic materials. They cannot be stored or used for more than a few hours or days.

Thus, we feel that both phantom types have limitations which prevent their use as standardized test systems. Although physical phantoms produce more realistic EIT signals than their mesh counterparts, there are neither physical phantoms nor traceable and well-defined test objects to perform system calibrations or evaluations under reproducible conditions. This work was motivated by the clear need for tools and systematic procedures to evaluate the performance and image quality of current and future EIT systems.

In order to quantitatively and reproducibly validate EIT system performance, we propose a reproducible evaluation procedure as well as methodology for image and data analysis using a phantom in conjunction with electrically and geometrically characterized traceable test objects. We envision the components in system performance evaluation as illustrated in figure 1. For an EIT system, consisting of an electrode interface, EIT stimulation and measurement hardware and an image reconstruction algorithm, measurements are performed using a specified measurement protocol and the data are systematically analysed by performance analysis algorithm. We describe the detailed procedure for each evaluation step, and then illustrate the analysis for three EIT systems. In order to facilitate use of this test protocol, all hardware and software components are made publically available. 


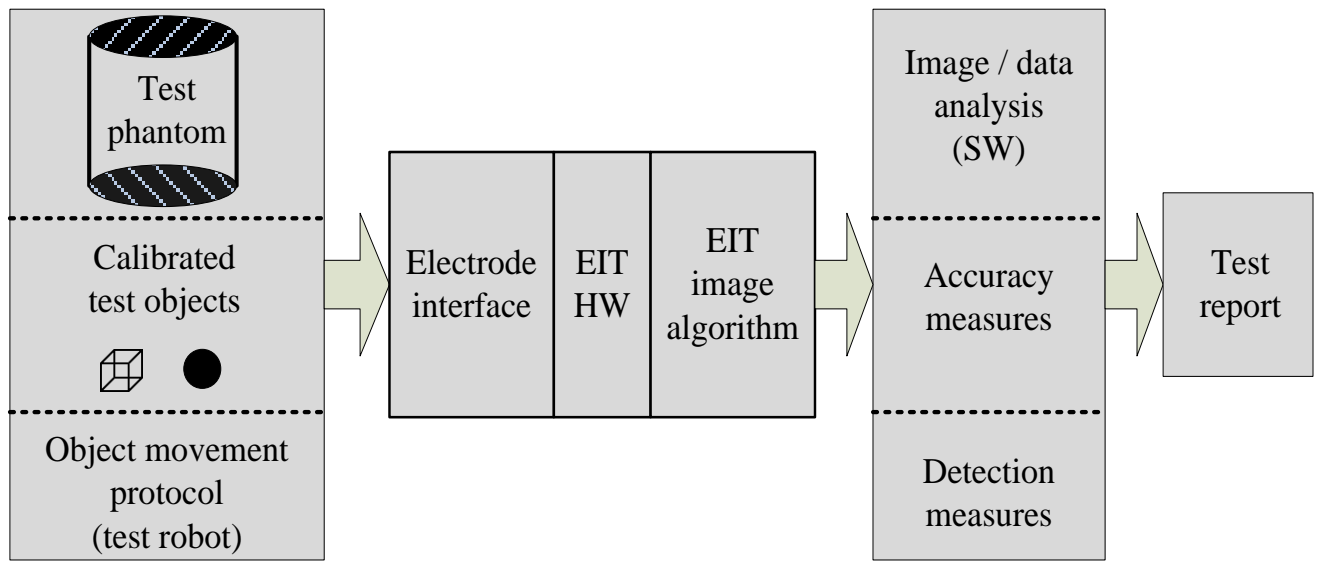

Figure 1. Schematic diagram of proposed test setup and procedure to evaluate EIT system performance.

\section{Evaluation Methodology}

\subsection{Evaluation overview}

Given an EIT measurement system, including body interface (i.e. cables and electrodes), electronic stimulation and measurement hardware, and image reconstruction software, we evaluate the system as illustrated in figure 1 . We first give an overview of the procedure and describe the evaluation goals. The EIT system is connected to the phantom, and three sets of measurements are performed: 1) homogeneous tank measurements, designed to evaluate system noise and accuracy, 2) measurements with one conductivity contrast which is moved horizontally at several vertical offsets from the electrode plane, designed to measure amplitude response, position accuracy, resolution, and detectability limits; and 3) measurements with two conductivity contrasts, designed to measure limits of distinguishability of contrasts. From these measurements, EIT images are reconstructed, and these images are then analysed using the described procedure to calculate the parameters shown in figure 2. Measurements are analysed in three ways. First, measured data are analysed in terms of noise, accuracy, drift and reciprocity accuracy (block $A$ in figure 2). Next, from reconstructed images, the reliability of EIT detection of a single targets, and of distinguishing two targets is determined (block $B$ ). These measures depend both on the image contrast strength and image noise (as determined from multiple measures). Finally, measures of image reconstruction accuracy (block $C$ ) based on those defined for GREIT (Adler et al 2009) are calculated (based on mean reconstructed images for each position).

\subsection{EIT measurement protocol}

The following measurement protocol requires an EIT system and a cylindrical tank filled with conductive saline with tank electrode connections for each EIT electrode. The tank radius is $r_{0}$ and the vertical centre of the electrode placements is defined to 


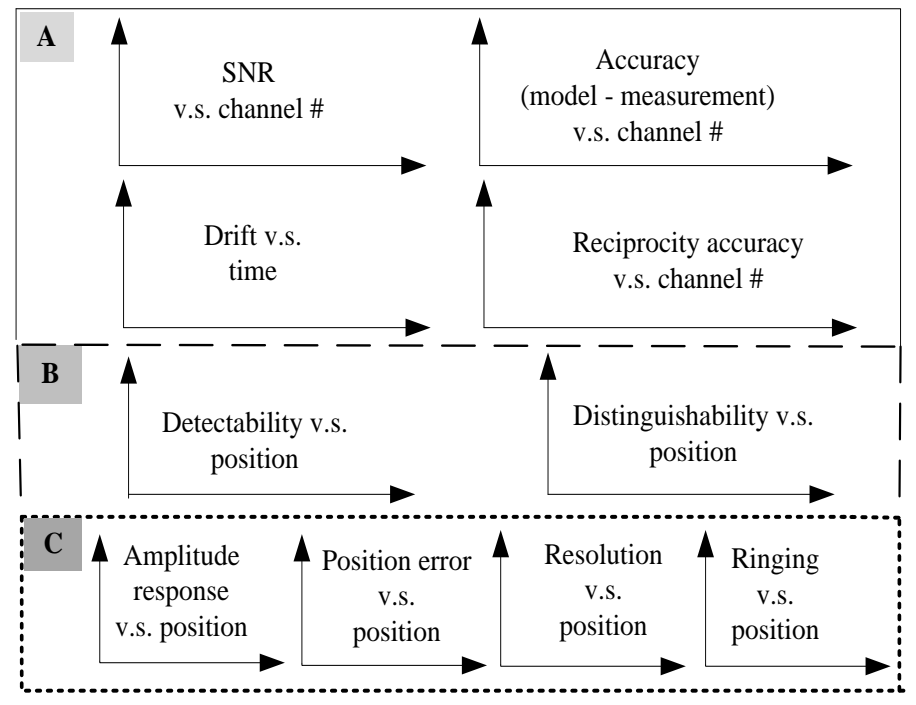

Figure 2. Concept diagram of evaluation of EIT system performance, where $A$ block is for noise and accuracy; $B$ block is for detectability of a single target and distinguishability of two targets; $C$ block is image reconstruction accuracy.

be $Z=0$. Using $N_{e}$ electrodes (our systems have $N_{e}=16$ ), a sequence frames, $\mathbf{v}$, of EIT data are acquired by repeated measurement, where each frame contains a complete set of current stimulation and measurement. Each individual pattern of measurement is called a measurement channel, such that channel $i$ is $[\mathbf{v}]_{i}$ (for a system which does not measure on stimulated electrodes, there are typically $N e \times(N e-3)$ channels $)$. Three conductivity contrasting test objects (one of volume $V_{T}$ and two of volume $\frac{1}{2} V_{T}$ ) are required. A target placed with its centre at a radial position of $r_{t}$ is defined to have a normalized radial position, $\hat{r}=r_{t} / r_{0}$. We use a saline tank of $28 \mathrm{~cm}$ diameter and $36 \mathrm{~cm}$ height, with saline of conductivity $0.8 \mathrm{~S} / \mathrm{m}$ filled to a height of $30 \mathrm{~cm}$ with a single electrode plane placed so the depth of saline above and below the electrode plane is equal. Holes for electrodes are drilled into the acrylic tank and flush filled with cylindrical gold plated electrodes with diameter $2.8 \mathrm{~mm}$. Electrodes have electrical connections to which the EIT system cabling is connected. Plastic non-conductive cubic targets are used so $V_{T}=100 \mathrm{ml}$.

In order to ensure accurate measurements, it is important to ensure that: the temperature of the saline is kept constant, since saline conductivities vary strongly with temperature (Fofonoff and Millard 1982), and target placements be done accurately and slowly enough that no ripples are created on the liquid surface. The following three measurement protocols are defined (corresponding to the blocks in figure 2), and illustrated in figure 3 (including an image of the phantom system and position controller used in this paper).

A Homogeneous tank measurements: In the homogeneous saline-filled tank, an acquisition of a sequence of at least 300 frames of data is performed. This acquisition is then sequentially repeated at delay times $\mathrm{T}, 2 \mathrm{~T} \ldots \mathrm{nT}$ for a defined delay, $\mathrm{T}$, 
until at least one hour has elapsed. We use a delay of 5 minutes and acquire data for 2 hours.

B Single conductivity contrast targets: A single conductivity contrasting target of volume, $V_{T}$, is placed in the tank and moved through a sequence of horizontal positions at a number of different vertical planes. First, an empty (homogeneous) tank measurement sequence is performed (as a reference for difference imaging), with at least 10 frames. Next, for each tested vertical plane, the target is moved from a position near the edge to the centre and back to a different edge in steps. At each target position step, first a delay is made to ensure that any disturbance at the saline surface dissipates, and then at least 10 EIT frames are acquired. The justification for the movement to and from the centre is to test for any rotational asymmetry in the EIT test response. We use horizontal positions separated by $2 \mathrm{~cm}$ and vertical positions of $Z=0$ and $Z=7 \mathrm{~cm}$. The target is moved in a horizontal line from a position where the target almost touches the electrodes to a mirror image position on the opposite side, as illustrated in figure 3(b). The conceptual framework for analysis of these data is given in figure 5(a).

C Two conductivity contrast targets: In order to perform measures of the distinguishability and resolution, two targets are placed in the tank as shown in figure 3(c). Each test is performed as follows: a target of volume, $V_{T}$, is placed at a given position and at least 10 frames are acquired. Next, the target is removed, and two targets of $\frac{1}{2} V_{T}$ are placed at the same position and moved apart while at least 10 frames are acquired at each movement step. We place a $100 \mathrm{ml}$ target position in the centre at $Z=0$, and move two $50 \mathrm{ml}$ targets horizontally apart at spaces of $2 \mathrm{~cm}$. The conceptual framework for analysis of these data is given in figure $5(\mathrm{~b})$.

\subsection{Performance parameters}

Based on the measurements performed in the previous section, a set of evaluation parameters is calculated as defined in this section, characterizing: a) data and image noise, and data accuracy, b) detectability and distinguishability of single and multiple contrasts, and c) accuracy of reconstructed images. The calculations for each parameter are described below, and each calculation is labelled corresponding to the block in the concept diagram (figure 2), such that B2 is the $2^{\text {nd }}$ block in section $B$. The accuracy calculations (a) are motivated by those of Gagnon et al (2008) and Hahn et al (2000), and the calculations of (c) by Weeler et al (2002) and Adler et al (2009). To measure image reconstruction accuracy, we use figures of merit motivated by the GREIT algorithm (Adler et al 2009) (C1) amplitude response (AR), (C2) position error (PE), (C3) resolution (RES), and (C4) ringing (RNG). The calculation of these figures of merit is illustrated in figure 4. To facilitate use of these calculations, we have contributed the software to perform these calculations as part of EIDORS (Adler and Lionheart 2006).

A1 Signal to Noise Ratio (SNR): The SNR estimates the precision ( or reproducibility) of measurement, which quantifies the degree to which repeated measurements under 


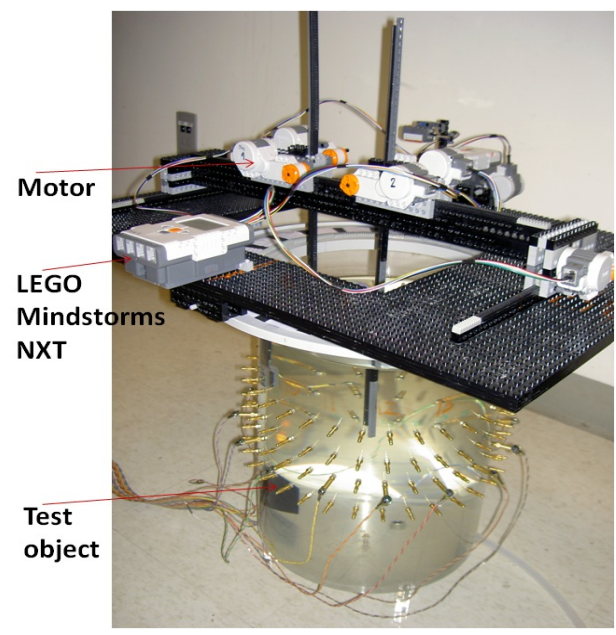

(a)

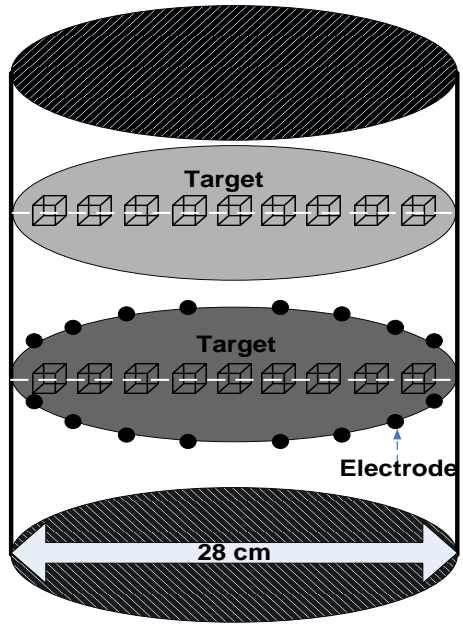

(b)

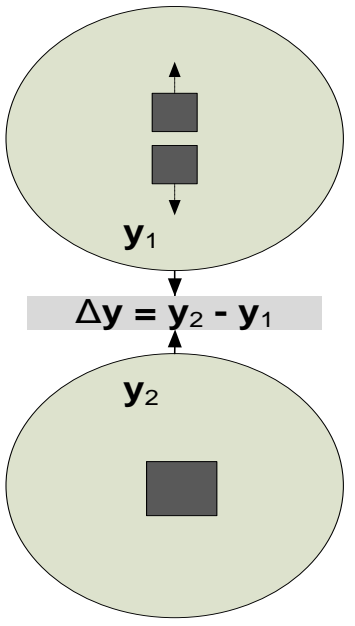

(c)

Figure 3. (a) Photo of the phantom and position controller; (b) Schematic drawing of movement protocols - a horizontal movement of a single object in two planes separately (3D view); (c) horizontal movement of two cubes of $50 \mathrm{ml}$ (top) away from the center to the edge and a cube of $100 \mathrm{ml}$ (bottom) fixed in the center of the tank (2D view).
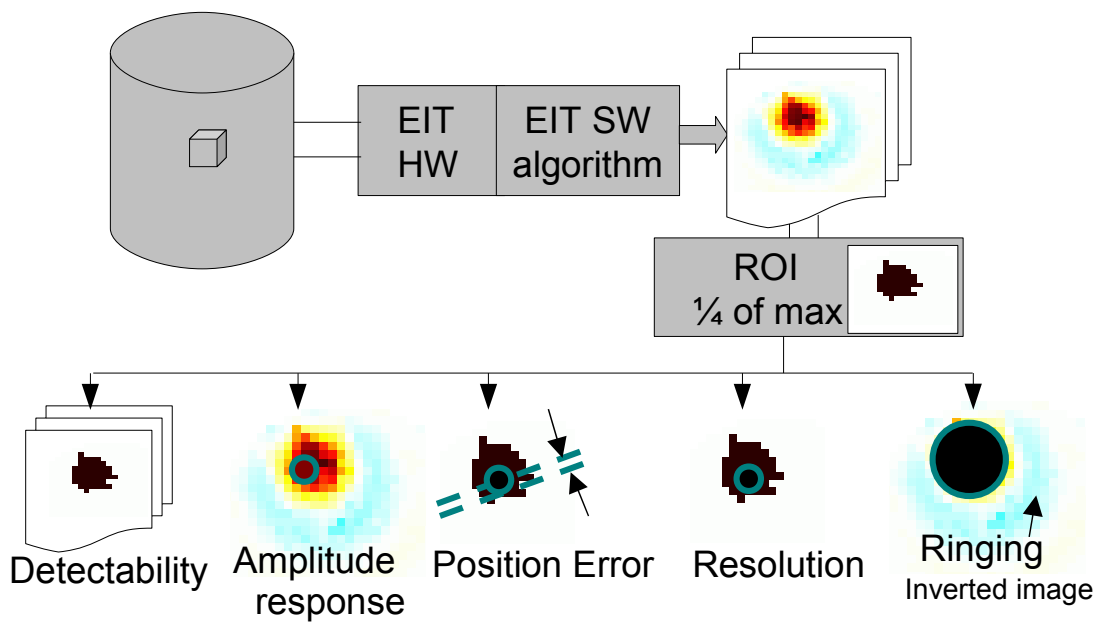

Figure 4. Procedures for analyzing image data: first row- phantom system, EIT measurement system and reconstruction algorithm, and $\mathrm{N}$ frames of the reconstructed images; second row: calculation of ROI over one-fourth amplitude set; third row: calculation of $z$ and GREIT parameters (Adler et al 2009).

unchanged conditions show the same results. The SNR is estimated classically from the ratio of mean signal to noise level for each of the measurement channels:

$$
\mathrm{SNR}_{i}=\frac{[\overline{\mathbf{v}}]_{i}}{\mathrm{SD}_{[\mathbf{V}]_{i}}}
$$

where $\mathrm{SD}_{\mathrm{v}_{i}}$ is the noise amplitude, obtained using standard deviation of multiple measurements for each channel and $[\overline{\mathbf{v}}]_{i}$ is signal amplitude, estimated using the mean value of the multiple measurements for each channel. 
A2 Accuracy (AC): The accuracy of a measurement system describes the closeness of measured quantities to their true value. In this case, the "true" value is assumed to be that generated by a numerical model of the homogeneous cylindrical tank for which both finite element model (FEM) and analytic (Kleinermann et al 2000) exist. We use a 3D FEM with sufficient node density such that relative voltage errors were below $0.05 \%$. This threshold was chosen to exceed the capabilities of the systems to which we have access. The FEM was determined by successively decreasing the maximum allowed node spacing and comparing a very fine model. A FEM of $380 \mathrm{k}$ elements and $72 \mathrm{k}$ nodes was required for the relative voltage difference to the fine model to decrease below the threshold. The FEM simulation, $\mathbf{v}^{(\text {sim })}$, is scaled by a linear constant to best fit the measured data, $\mathbf{v}$. The accuracy of $i^{\text {th }}$ channel is

$$
\mathrm{AC}_{i}=\left(1-\left|[\overline{\mathbf{v}}]_{i}-\left[\mathbf{v}^{(\operatorname{sim})}\right]_{i}\right|\right) \times 100 \%
$$

where $[\overline{\mathbf{v}}]_{i}$ is the mean homogeneous tank measurement for channel $i$. Measurements which accurately fit to the FEM have $\mathrm{AC}=100 \%$, while values with poor fit have lower values. The simulated voltages are normalized to give a best fit to the real measurement. Average accuracy is the mean of all channels. This measure corresponds to the Modelling Accuracy measure of Gagnon et al (2008).

A3 Drift is a measure of the change in average value of measurements over time. Such changes occur in EIT due to changes in electrode properties and the temperature changes of the EIT hardware. Since drift will also occur due to changes in saline temperature, this must be carefully controlled. We measure drift using the Allan Variance (Allan 1987), $\mathrm{AV}_{v}^{2}(\tau)$, which characterizes the stability of systems as a function of the averaging time $\tau$. The Allan deviation $\operatorname{AV}_{v}(\tau)$ is the square root of the Allan variance. Data are divided into bins based on an averaging time $\tau$ and averaged in each bin $(i)$, yielding an average $\overline{\mathbf{v}}_{i}$. The Allan Variance $\left(\operatorname{AV}_{v}^{2}(\tau)\right)$ can be written as a function of the averaging time $\tau$ (Allan 1987):

$$
\mathrm{AV}_{v}^{2}(\tau)=\frac{1}{2(n-1)} \sum_{i}\left(\overline{\mathbf{v}}(\tau)_{i+1}-\overline{\mathbf{v}}(\tau)_{i}\right)^{2}
$$

where $n$ is the total number of bins, $\overline{\mathbf{v}}_{i}$ is the average value of the measurement in bin $i$. For increasing $\tau$, the variance decreases and would be expected to converge for a system with no drift. These systematic errors cause AV to increase again. Thus, AV characterizes the relationship between system noise and drift over the measured period.

A4 Reciprocity Accuracy (RA): The reciprocity principle (Plonsey 1963) indicates that EIT measurements from a stimulation-measurement pair should not change if the current stimulation and voltage measurement electrodes are swapped. Using this identity, we can compare all EIT measurements to those from the corresponding reciprocal pair. If the measures values match, the values are most likely accurate. Any differences must be explained by errors and noise in data acquisition in one or 
both measurements. The RA of $i^{\text {th }}$ measurement sequence is calculated as

$$
\mathrm{RA}_{i}=\left(1-\frac{\left|[\overline{\mathbf{v}}]_{i}-[\overline{\mathbf{v}}]_{r(i)}\right|}{\left|[\overline{\mathbf{v}}]_{i}\right|}\right) \times 100 \%
$$

where $[\overline{\mathbf{v}}]_{i}$ is the mean tank measurement for channel $i$, and $[\overline{\mathbf{v}}]_{r(i)}$ is the mean measurement on the channel $r(i)$ which contains the reciprocal electrode configuration to channel $i$. Measurements that accurately match the reciprocal measurements $\left(\overline{\mathbf{v}}_{i}=\overline{\mathbf{v}}_{r(i)}\right)$ have the $\mathrm{RA}=100 \%$, while values with poor match have lower values. This measure is related to the reciprocity error proposed by Riu and Anton (2010) who noted that the error varies with EIT system frequency and electrode contact.

B1 Detectability is a measure of the ability of EIT to reliably detect targets within a body. The measure may be posed as a hypothesis test, in which EIT measures are used to calculate a $z$ statistic by which to distinguish the presence of a target from the null hypothesis (homogeneous tank), where $z$ is a measure of the EIT system performance

$$
z=\frac{\hat{\mathbf{x}}_{q}}{s}
$$

The detectability is thus designed to indicate the amplitude of the imaged target region and SNR. A conceptual diagram of the detectability procedure is shown in figure 5(a) to detect one object. The signal amplitude, $\hat{\mathbf{x}}_{q}$, is the mean image in a ROI, calculated from multiple reconstructed images (figure 4). $s$ represents the EIT noise amplitude, calculated from the standard deviation of multiple reconstructed images. Here, the ROI is defined as image amplitude greater than $\frac{1}{4}$ of the maximum value. It includes the region of the reconstructed image which represents the largest contribution to the image amplitude. A ROI is selected (rather than the entire image) to avoid contamination of the noise region by image artefacts outside the ROI. The detectability and distinguishability measures are based on the analysis of Isaacson et al (1986) and our adaptations of it (Adler et al 2010). However, we prefer to reserve the term distinguishability for the ability to separate two targets from one, while using detectability for the single object detection task.

B2 Distinguishability of two objects is a measure of the ability of EIT to reliably distinguish structural details of targets, and specifically whether the same volume target is present as two objects or one. This concept is illustrated in figure $5(\mathrm{~b})$ using difference EIT measurements from one target of volume $V_{T}$ and those from two targets representing the same volume $\frac{1}{2} V_{T}$ as a function of the target separation, using the protocol figure 3(c). Using these data, the identical calculation (5) is performed. We determine the resolution as the full width at $\frac{1}{4}$ maximum of the reconstructed image amplitude. 


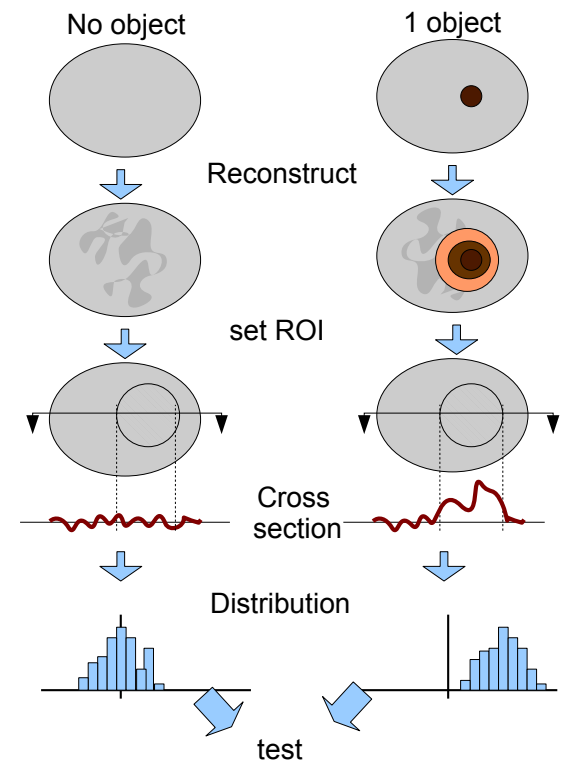

(a)

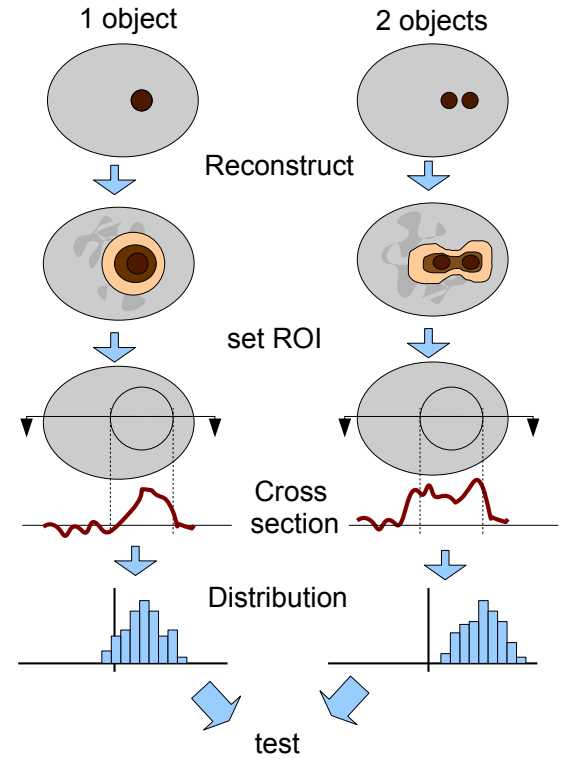

(b)

Figure 5. A conceptual diagram of detectability and distinguishability showing procedures for detecting one or two targets: (a) detectability test to detect one object, (b) distinguishability test to distinguish one from two targets.

C1 Amplitude Response $(A R)$ is used to measure the ratio of image pixel amplitude in the target v.s. target position.

$$
\mathrm{AR}=\frac{1}{V_{T}} \sum[\hat{\mathbf{x}}]_{k}
$$

where $V_{T}$ is an original target volume used for normalization and we sum over the entire reconstructed image, $\hat{\mathbf{x}}$, since all image amplitude is considered to be part of the amplitude response. AR indicates how targets at different positions contribute to overall image amplitude. It should ideally be uniform over the range of regions of interest.

C2 Position Error (PE) measures the mismatch between the reconstructed image centre and the original position of the target.

$$
\mathrm{PE}=r_{t}-r_{q}
$$

where $r_{t}$ is the target position and $r_{q}$ is the center of gravity (CoG) of $\hat{\mathbf{x}}_{q}$. The significance of $\mathrm{PE}$ is that such errors may lead to unreliable interpretation of the images.

C3 Resolution (RES) is a measure of the smallest visible object within a system. RES is defined as the sum of reconstructed images on the region of interest to total reconstructed image. By taking the square root, RES measures radius ratios rather than area ratios, which can be written as:

$$
\mathrm{RES}=\sqrt{A_{q} / A_{0}}
$$


where $A_{q}=\sum\left[\hat{\mathbf{x}}_{q}\right]_{k}$, the number of pixels in $\hat{\mathbf{x}}_{q}$ and $A_{0}$ is the area (in pixels) of the entire reconstructed medium. The system resolution is reflected both in the RES measure and in the distinguishability of two objects. In the latter case, it also reflects the system noise performance.

C4 Ringing ( $R N G$ ) measures whether reconstructed images show areas of opposite sign surrounding the main reconstructed target area. For a circle $C$ centred at the $\mathrm{CoG}$ of $\hat{\mathbf{x}}_{q}$, we define

$$
\mathrm{RNG}=\frac{A_{\text {out }}}{A_{\text {in }}}
$$

where $A_{\text {in }}$ is the image, $\hat{\mathbf{x}}$, content within $C$, and $A_{\text {out }}$ is the image content outside $C$ and of inverse sign to the contrast.

\section{Evaluation system}

We have designed and implemented the test system and protocol defined in the previous section. The test protocol for a single EIT system is described.

Phantom and test objects: Tests were performed using a saline filled plexiglas tank, as shown in figure 3(a). Saline conductivity was measured and monitored using a conductivity meter ECTestr (Oakton Instruments, USA). We used non-conductive plastic cubes with two different volumes, $50 \mathrm{ml}$ (side length of $3.6 \mathrm{~cm}$ ) and $100 \mathrm{ml}$ (side

length of $4.3 \mathrm{~cm}$ ) with a resistivity value of much greater than $1 \mathrm{M} \Omega \cdot \mathrm{cm}^{-1}$ (Precision LCR Meter LCR-821, GW Instek, Korea). In order to avoid noise caused by water and the subsequent object motion, measurements were initiated with a $15 \mathrm{~s}$ delay after a target position was reached. The delay time was experimentally determined in pilot studies using camera and an light styrofoam boat with an indicator sail to exaggerate the motion signals.

Position controller system: To precisely and reproducibly position test objects within the saline tank a position controller was constructed from standard LEGO parts in connection with a set of motors and two controllers (LEGO NXT Mindstorm). Two test objects could be positioned independently by a total of six position controllers, two for each of the $X, Y$ and $Z$ directions. In order to overcome the limitations in the range of motion imposed by the physical size of the LEGO parts the entire control unit rotates $360^{\circ}$ on a plastic support placed on top of the cylindrical tank as shown in figure 3(a). The movement protocol was stored on a computer and transmitted to the position controllers via Bluetooth. A control program stores the coordinates and the position controller automatically moves the object to the desired locations. Accuracy and precision of test object center point positions were verified and deviations were less than $2 \mathrm{~mm}$ in the $X Y$-plane and less than $1 \mathrm{~mm}$ in the vertical $Z$-direction. EIT data acquisition was initiated manually after each robot positioning of the target, since it was not possible to reliably interface the robot controller with each EIT acquisition software system. 
EIT systems: Performance analysis was performed for three different EIT systems to which we have access. Each system has 16 electrodes and implements pair drive injection and measurement protocols. Raw data from the EIT systems is saved to disk, and a linear difference EIT image reconstruction is implemented. While the proposed evaluation scheme clearly provides a way to compare systems, it is not the goal of this paper to report on specific EIT system performance. Each EIT system was tested using adjacent stimulation and measurement (the Sheffield protocol from Brown and Seagar 1987), and was used with the provided electrode cables which varied in length from $0.5 \mathrm{~m}$ to $2 \mathrm{~m}$. The cabling type also varied between systems, with one of each system using simple wire, twisted wire and coaxial cable. In order to determine the test-retest accuracy, one system was tested three times, with complete replacement of tank contents between tests. This system had also been tested earlier, before an upgrade of the analog front-end electronics.

EIT algorithms: EIT reconstruction is performed with the linear time difference reconstruction algorithm as

$$
\hat{\mathbf{x}}=\left(\mathbf{J}^{T} \mathbf{J}+\lambda^{2} \mathbf{R}\right)^{-1} \mathbf{J}^{T} \mathbf{y}
$$

where $\mathbf{J}$ is the Jacobian. The regularization matrices $(\mathbf{R})$ is based on the discrete laplacian and regularization parameter $\lambda=0.15$. $\mathbf{y}$ is the normalized difference measurement vector: $[\mathbf{y}]_{i}=\left([\mathbf{v}]_{i}-\left[\mathbf{v}_{r}\right]_{i}\right) /\left[\mathbf{v}_{r}\right]_{i}$, where $\mathbf{v}_{r}$ is the reference measurements and $\mathbf{v}$ is the measured voltages. This algorithm is a one-step Gauss Newton linear difference solver, which has been used in many EIT implementations (e.g. Adler and Guardo 1996). One of the goals of the evaluation system is to measure the effect of the image reconstruction algorithm with the EIT measurement system. For these results, we wanted to avoid seeing effects specific to the algorithm, especially in comparing EIT systems, and thus selected a baseline reconstruction algorithm for all systems.

\section{Results}

The results are presented in two stages: stage one (figure 6) focuses on the evaluation of a single system performance using the conceptual performance measures defined in figure 2; stage two (figure 7) further extends the evaluation protocol for comparing performances of different EIT systems (of 3 available systems in this study) using only one representative measure from each protocol. Overall, it takes approximately 3 hours to perform all measurements after setting up the system. Of this time, approximately 2 hours are spent making measurements related to drift calculations.

Figure 6 shows measurement results for the evaluation of a single system performance. Three sets of results are obtained in terms of 1) noise, accuracy, drift and in figure 6(A) from the measured (homogeneous tank) data, 2) the detectability of a single target and distinguishability of two targets in figure 6(B), and 3) image reconstruction accuracy from a single target measurement using AR, PE, RES and $\mathrm{RNG}$ in figure $6(\mathrm{C})$ respectively. 

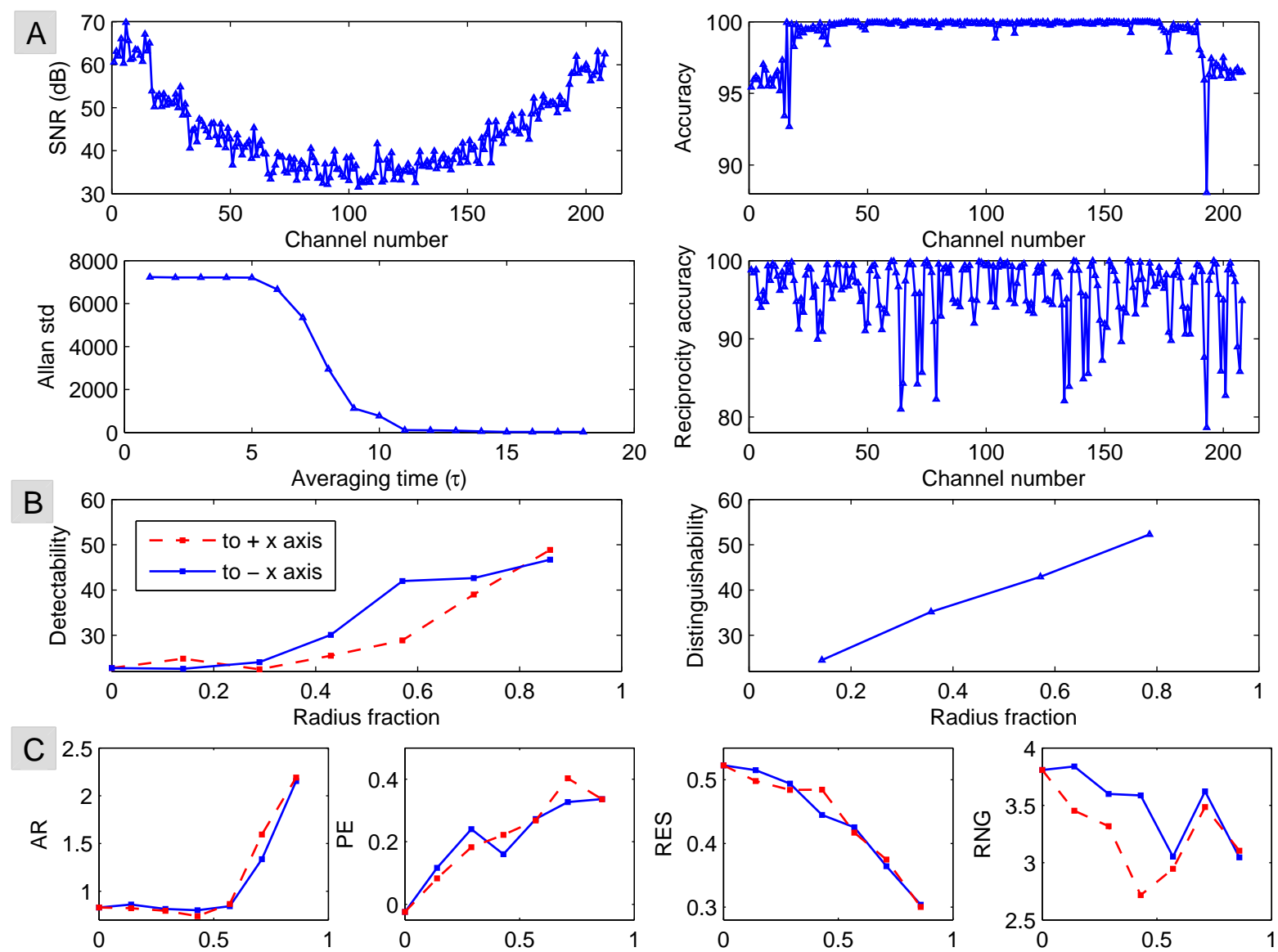

Figure 6. The evaluation of a single system performance using the performance figures of merit as A: SNR, accuracy, Allan deviation (std) and reciprocity accuracy; $\mathrm{B}$ : detectability and distinguishability; C: AR, PE, RES and RNG. "To $\pm \mathrm{X}$ axis" in the legend is used to indicate the direction of target displacement along $\mathrm{X}$ axis.

Results in figure 6(A1) show the performance of EIT system A based on SNR with the behaviour of specific channels with varying noise levels, figure 6(A2) shows a high level of accuracy with an average accuracy of $99.2 \%$ for this system. Figure 6(A3) shows the drift analysis of the system using the AV parameter. During the interval shown, AV decreases with increasing averaging time $(\tau)$ and does not subsequently increase, indicating that the system does not drift significantly within the measured period.

Figure 6(A4) shows the RA of the system based on two reciprocal measurements. RA fluctuates within one measurement period which indicate that the system does have certain reciprocity errors with an average RA of $96 \%$.

As expected (figure 6(B1)) large detectability values are observed when the single target move close to the edge of the tank, while values are less for targets in the centre. For the two-target distinguishability figure 6(B2), the values increase when the objects move further away from each other. In figure $6(\mathrm{C} 1, \mathrm{C} 2), \mathrm{AR}$ and $\mathrm{PE}$ increases as the target object gets closer to the boundary, especially high at the edge where the target was touched to the electrode (a case intentionally selected as the worst scenario). The 
values of RNG fluctuate in figure 6(C4) for the target close to the boundary of the tank.

Performance comparisons: Figure 7 shows performance comparisons of three EIT systems in terms of accuracy, detectability and image reconstruction accuracy (AR). The results show the behaviour and variations of accuracy and detectability for 3 systems. It can be seen from figure $7(\mathrm{a})$ that system $\mathrm{C}$ has larger and repetitive measurement errors. The average accuracy percentage of three systems (A, B and C) are 99.2, 98.4 and 87 respectively. Figure $7(\mathrm{~b})$ clearly gauges the performance of 3 systems in terms of the detectability of a single conductivity contrast targets moving from centre to the periphery of the tank.

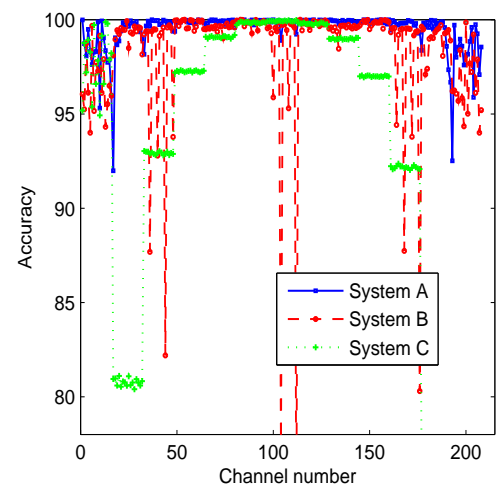

(a)

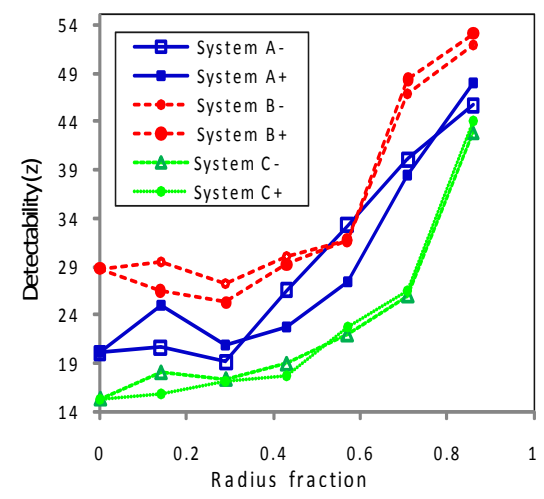

(b)

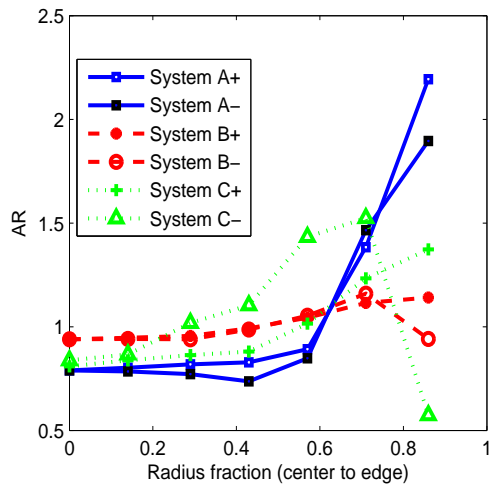

(c)

Figure 7. The performance comparison of three EIT systems in terms of accuracy (Left), detectability (Middle) and AR (Right) with the systems $A$ (blue solid line with square marker type), $B$ (red dashed line with asterisk marker type) and $C$ (green dotted line with plus marker type). " \pm " in the legend is used to indicate the direction of target displacement along $\mathrm{X}$ axis.

Although not the primary goal of this paper, it is also possible to use this system to compare different EIT systems in terms of noise, accuracy and image reconstruction performance. Three different systems are compared and a selection of performance parameters are shown in figure 7. In figure 7(a), the accuracy of A exceeds B and C. The relationship is similar in figure 7(b) where the detectability is shown to be largest in A and least in C. In figure 7(c), AR of all systems are more sensitive to conductivity changes close to the electrodes, while response is more stable for targets in the middle region of the tank.

One key feature of a reliable test system is a high test-retest repeatability. This value was calculated by repeating a complete test with the same system three times over two days, including replacement of tank saline. Figure 8 shows results for SNR and accuracy for three tests, as well as for an earlier test with the same system, before an analog hardware upgrade. Average channel SNR values were $44.2 \mathrm{~dB}$ (pre-upgrade) and $56.4 \mathrm{~dB}, 56.5 \mathrm{~dB}$ and $54.4 \mathrm{~dB}$, respectively (post-upgrade), showing a significant improvement due to the hardware change. The corresponding average accuracy (AC) values were $99.2 \%$ (pre-upgrade) and $98.6 \%, 98.7 \%$ and $98.7 \%$ (post-upgrade), did not 
show improvement. The repeatability of the measures for the same (post-upgrade) system were to within $0.05 \%$ accuracy and $2 \mathrm{~dB}$ SNR, which suggests the phantom system has a good test-retest repeatability.

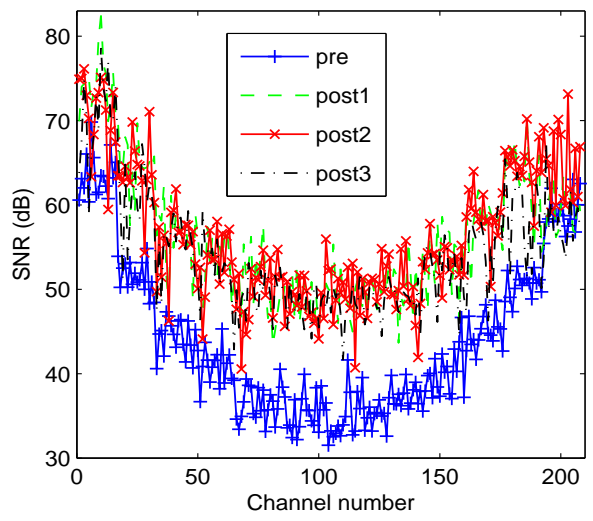

Figure 8. Test-retest comparison of EIT systems. SNR as a function of channel number is shown for the system before an analog hardware upgrade (pre) and for three repeated measures after upgrade (post1...3).

\section{Discussion}

In this paper, we proposed a phantom system design (figure 1) and test methodology (figure 2) based on reproducible procedures with characterized and traceable test objects to evaluate the performance of an EIT system. We showed that the proposed approach is also suitable for comparing different system performances and evaluating hardware improvements. Three sets of measurement protocols (figure 2) are defined: a homogenous tank measurement to analyze SNR, accuracy and drift, a detectability of a single target and distinguishability of two targets, and image reconstruction accuracy to give an indication on how accurate an EIT system is in determining image reconstruction accuracy in the tank. Extended comparisons are carried out on three EIT systems based on accuracy of measured data, detectability measures and AR for the image reconstruction accuracy.

For homogenous tank measurement, four measures (SNR, accuracy, drift and reciprocity accuracy) are used to analyze characteristics of an EIT system. The distinguishability and detectability are similar to SNR, where the distinguishability and detectability values at a location measure noise and resolution, and used for maximizing SNR. The position of two objects strongly affected the image reconstruction where two closely positioned objects look like a bigger single object, and the distinguishability value increases with the distance between the two objects. The results of image reconstruction accuracy based on AR, PE, RES and RNG showed that EIT systems are more sensitive to conductivity changes close to the electrodes.

We have attempted to make as few selections of arbitrary thresholds as possible 
in the elaboration of this test approach, so that, to the extent possible, factors in the EIT hardware and image calculations are reflected in the calculated measures. The

use of a ROI based on a $\frac{1}{4}$ threshold of the maximum value has the advantage that low amplitude noise far from the ROI will be rejected. If the noise amplitude is larger than this threshold, it will be retained and will be seen in the results. For our targets, we select cubic objects over spheres because flat sided targets can be placed flush to allow two objects in the distinguishability protocol to better approximate one object when touching. Another benefit of cubic targets is the ease of testing the conductivity properties in all axes, and thereby testing for anisotropy. We choose to use very resistive targets which provide a high conductivity contrast, since targets with low conductivity contrast are more difficult to accurately manufacture, and require significantly more experimental care on tank temperature and salinity to ensure the accurate contrast value. The value of the test results for characterization of the EIT system is not significantly enhanced by use of low contrast targets. The contact impedance of the electrode-saline connection in a tank phantom is generally much lower (and less variable) than the electrode-skin interface in EIT practice. However, the magnitude of sources of data corruption, such as common-mode noise and electromagnetic interference, depends on the contact impedance level. This means that saline tanks cannot adequately model the effects of the electrode-skin interface. At the same time, we note that electrode-skin effects and its impact on EIT data are still poorly understood.

Although SNR is commonly used in EIT community to measure EIT system performance, the value may vary according to test conditions and how capacity of the system is used. Interestingly, all three systems have very similar average SNR value (44.2, 44.5 and $45.9 \mathrm{~dB}$ for systems $\mathrm{A}, \mathrm{B}$ and $\mathrm{C}$ respectively), but the three systems clearly perform differently in our calculations using accuracy, detectability and AR as shown in figure 7 . Such results allow us to evaluate relative system performance, although this is not our main objective in this study.

The phantom based test methodology is found to be effective and useful to evaluate EIT system performance. Moreover, we can compare the performance of different EIT hardware and image reconstruction systems. Also, in addition to a use for comparing and evaluating different system performance, it can provides developers a test standard against which hardware improvements as well as new system developments can be tested. To encourage use of this methodology, we have made public all details of the test system, including software to measure performance parameters, instructions on the design of the (LEGO) position controller and phantom construction details on eidors3d.sf.net/data_contrib.shtml under an open source license.

\section{Acknowledgments}

The authors would like to thank to Sascha L. Reidt from CSEM Landquart for designing, building and supporting the position controller and Obaydah Jaber from Carleton University for his help with experiments. This work was partially funded by a grant 
from NSERC Canada.

\section{References}

Adler A, Gaggero P and Maimaitijiang Y, Distinguishability in EIT using a hypothesistesting model, Int. Conf. Electrical Bio-Impedance and Electrical Impedance Tomography, Gainville, Fl, USA, 4-8 April

Adler A, et al 2009 GREIT: a unified approach to 2D linear EIT reconstruction of lung images Physiol. Meas. 30 S35-S55

Adler A and Lionheart WR 2006 Uses and abuses of EIDORS: An extensible software base for EIT", Physiol. Meas. 27 S25-S42.

Adler A and Guardo R 1996 Electrical impedance tomography: regularised imaging and contrast detec- tion, IEEE Trans. Med. Imaging 15 170-179.

Allan D W, Time and frequency (time domain) characterization, estimation and prediction of precision clocks and oscillators, IEEE T Ultrason Ferroelect Freq Contr 34: Nov. 1987.

Brown B H and Seagar A D 1987 The Sheffield data collection system, Clin. Phys. Physiol. Meas. 8, Suppl. A, 91-97.

Fofonoff N P and Millard R C, Algorithms for computation of fundamental properties of seawater, Unesco Technical Papers in Marine Science, Vol. 44 (1982), p. 53.

Frerichs I, Dargaville PA, Dudykevych T et al. (2003) Electrical impedance tomography: a method for monitoring regional lung aeration and tidal volume distribution? Intensive Care Med 29:2312-2316.

Gagnon H, Guardo R 2005 A method for designing electrical impedance tomography (EIT) phantoms of arbitrary shape and conductivity distribution Biomed. Tech. 50 (Suppl. 1) 297-298.

Gagnon H, Cousineau M, Adler A, Hartinger A 2010 A resistive mesh phantom for assessing the performance of EIT systems IEEE T Biomed. Eng. 57 (9):2257-2266, 2010.

Griffiths H 1988 A phantom for electrical impedance tomography Clin. Phys. Physiol. Meas. 9 (Suppl. A) 15-20.

Griffiths H 1995 A Cole phantom for EIT Physiol. Meas. 16 A29-38.

Hahn G, Beer M, Frerichs I, Dudykevych T, Schröder T and Hellige G 2000 A simple method to check the dynamic performance of electrical impedance tomography systems Physiol. Meas. 21 53-60.

Hahn G, Just A, Dittmar J, Hellige G 2008 Systematic errors of EIT systems determined by easily-scalable resistive phantoms Physiol. Meas. 29 S163-72.

Kleinermann F, Avis NF, Alhargan FA 2000 Analytical solution to the threedimensional electrical forward problem for a circular cylinder Inverse Problems 16 461-468.

Oh T I, Koo H, Lee K H, Kim S M, Lee J, Kim S W, Seo J K and Woo E J 2008 Validation of a multi-frequency electrical impedance tomography (mfEIT) system 
KHU Mark1: impedance spectroscopy and time-difference imaging Physiol. Meas. $29295-307$.

Rigaud B, Morucci J P 1996 Bioelectrical impedance techniques in medicine. Part III: Impedance imaging. First section: general concepts and hardware Crit. Rev. Biomed. Eng. 24 467-597.

Riu PJ, Anton D 2010 Performance assessment of EIT measurement systems J Physics: Conference Series 224:012015

Plonsey R 1963 Reciprocity applied to volume conductors and the ECG, IEEE T Biomed Eng, 10:9-12

Schneider I D, Kleffel R, Jennings D and Courtenay A J 2000 Design of an electrical impedance tomography phantom using active elements Med. Biol. Eng. Comput. 38 390-394.

Victorino JA, Borges JB, Okamoto VN, Matos GFJ, Tucci MR, Caramez MPR, Tanaka H, Sipmann FS, Santos DCB, Barbas CSV, Carvalho CRR, Amato MBP (2004) Imbalances in regional lung ventilation. A validation study on electrical impedance tomography. Am J Respir Crit Care Med 169:791-800.

Wheeler JL, Wang W, Tang M 2002 A comparison of methods for measurement of spatial resolution in two-dimensional circular EIT images Physiol. Meas. 23169 176. 